\title{
Oxygen Affinity of Transition Metal Cations: A Coherent Descriptor in Catalytic Oxygenate Transformations
}

\author{
Huixiang $\mathrm{Li}^{1}$, Peifang $\mathrm{Yan}^{1}$, B.Q. Xu ${ }^{1}$, and Conrad Zhang ${ }^{2}$ \\ ${ }^{1}$ Affiliation not available \\ ${ }^{2}$ Dalian Institute of Chemical Physics
}

September 24, 2021

\begin{abstract}
This work reports a complementary descriptor to acidity for metal cations, oxygen affinity, to scale the interaction strength of metal cations with oxygenate groups. A method for measuring oxygen affinity is developed in alkyl methylimidazolium chloride solvents using in-situ far infrared (FIR) spectroscopy. The relative oxygen affinity values of metal cations in metal chlorides with model compounds including cyclopentanol (for alcohols), cyclohexanone (for ketones), ethylene glycol diethyl ether (for ethers), and $\mathrm{H} 2 \mathrm{O}$ are measured from the dissociation kinetics of the metal-oxygenate complexes. The concentrations of metalchloride and metal-oxygenate complexes are determined during evaporative removal of oxygenate molecules according to the FIR absorbance of metal-Cl bonds. The oxygen affinity values are shown to reasonably account for the catalytic performances of metal cations in furfural-acetone condensation and glucose isomerization reactions. The oxygen affinity descriptor could be potentially exploited as an intrinsic property of metal cations broadly in metal chlorides and metal oxides.
\end{abstract}

\section{Hosted file}

Manuscript_Conrad Zhang.docx available at https://authorea.com/users/435031/articles/538335oxygen-affinity-of-transition-metal-cations-a-coherent-descriptor-in-catalytic-

oxygenate-transformations 